\title{
Moving Architecture, Animated Maze: The Intertextuality Between Player and Environment Agents
}

\author{
Yiou Wang* \\ Harvard University \\ Cambridge, Massachusetts, USA \\ yiouwang@gsd.harvard.edu
}

\author{
Yujie Wang* \\ Massachusetts Institute of Technology \\ Cambridge, Massachusetts, USA \\ yujiew@mit.edu
}

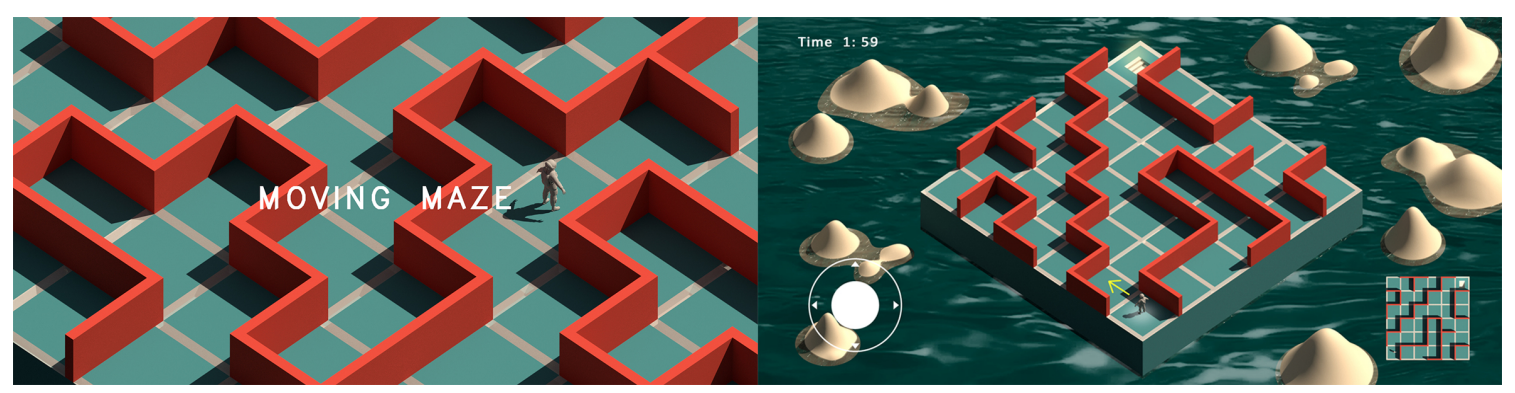

Figure 1: The landing scene and the game scene of Level 1.

\begin{abstract}
The maze, a classic architectural type of post-functional nature, is reinvented through the contemporary lens of video game. With novel analytical insights and computing methodologies on the system-unit relationship of a maze, we design and develop a Moving Maze that moves its parts methodically in response to the player's movement. A disorienting and adaptive system composed of identical parts, the Moving Maze is deconstructed into the nonsubdivisible unit, which can propagate into a field through replication and orthogonal rotation. The game generates unit-to-system interactive outcomes with fragmental movements using gamerrelational rules. In achieving difficulty progression and game balance through Reinforcement Learning, the maze arouses problemsolving curiosity and immerses the player in a risk-reward structure. Centering the game mechanics on interactivity and adaptability, we enhance player engagement in this cognitive puzzle game through balanced player and environment agency. An artwork synthesizing procedural computation with gaming architecture, the Moving Maze pushes the imaginative boundary of what a maze can be and embodies the philosophy that systemic complexities arise from the simplest elements.
\end{abstract}

\section{CCS CONCEPTS}

- Games/Play; • Design Method; • Storytelling;

Both authors contributed equally to this research.

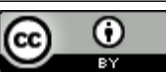

This work is licensed under a Creative Commons Attribution International 4.0 License.

C\&C '21, fune 22-23, 2021, Virtual Event, Italy (c) 2021 Copyright held by the owner/author(s). ACM ISBN 978-1-4503-8376-9/21/06.

https://doi.org/10.1145/3450741.3466806

\section{KEYWORDS}

Game Theory, Maze, Game Design, Generative System, Architecture, Reinforcement Learning

\section{ACM Reference Format:}

Yiou Wang and Yujie Wang. 2021. Moving Architecture, Animated Maze: The Intertextuality Between Player and Environment Agents. In Creativity and Cognition (C\&C '21), fune 22-23, 2021, Virtual Event, Italy. ACM, New York, NY, USA, 5 pages. https://doi.org/10.1145/3450741.3466806

\section{INTRODUCTION}

In a system, complexities arise from the simplest elements. In a game, the best system reveals the most scenarios with the least contrivance [1]. The maze is a common architectural type for play, but animating the walls endows the maze with heightened agency. In creating a maze that could move its parts in response to the player's movement, we design a method that generates an unpredictable complex system whose fragmental movement complicates the player's effort to find a solution, but adapts its intelligence to the player's level. Any standard maze is a disorienting system composed of repetitive parts. Ultimately, we are devising a computational system that reacts to the player's movement by intelligently choosing which parts to rotate and how. This maze is a system designed to generate temporal diversity of outcomes with the simplest principles. With each move the player makes, the interactive maze adjusts itself incessantly, while the player continues to engage in an intellectually inventive experience navigating amidst the disorder.

\section{A GAME APPROACH TO MAZES}

The maze intrigues people as an architectural gamescape with scarce programmatic utility. Derived from the ancient 2-D labyrinth, the maze elevated into a 3-D structure, relinquished its critical role in religious and symbolic affairs, and became a model for a complex risk-reward structure. What distinguishes multicursal mazes from labyrinths is the presence of choice - junctions that potentially 
lead to dead ends, which requires cognitive optimization to weigh different options [12]. The culture of architecture profoundly transformed since maze diverged from labyrinth from the Renaissance onto modernity, deviating from order and symbolism, reorienting toward public recreation which obscures social purposes and embraces a lightheartedness. The existence of multiple pathways leads to confusion and challenge, emotions that arouse the curiosity of routing [8], triggering an entertained psychological state of problem solving.

The maze reveals significant intertextuality with a puzzle game. The multicursal nature implies the indeterminate fate of the player and possible lurking dangers, characteristic of an adventure game. The narrow pathways and walls slightly exceeding human height provide two options of gaming the maze: an adventure option from a first-person perspective which immerses the player in limited local information and implies danger from unseen enemies, where success depends more on quick wit; the other, a puzzle option from an aerial perspective where success of an omniscient player depends more on strategic thinking. As a primarily puzzle game, a good maze rewards the player with intellectual joy of eureka moments as in go. To up the excitement and challenge to the conventional maze, we introduce maze movement in opposition to the player and the "enemy" of time: a time limit imposed to heighten the risk of wrong choice and increase the reward of correct ones.

\section{MAZE DESIGN}

\subsection{Component Analysis and Constructive Logic}

The maze system operates on generality and specificity: it can be deconstructed down to the same set of components at the fragment level, but the telling distinction is the node - whether it is a corner, a dead end, or a junction. To construct a maze means to construct the nodes, and merely fill in the rest with continuous passage . Echoing with our theory is the finding by Jeff Saward [10] that to transmit a labyrinth dots are drawn first and the remaining lines are simply connected to the dots, and the idea by Kappraff et al. [6] which identifies maze-making methods as node permutations of meander links and knots.

At the mesoscale, the maze consists of four linguistic archetypes that are structurally and functionally distinct - passage, and three nodes: corner, junction, and dead end. The four components construct the maze using logical organizing principles just as vocabulary constructs language using grammar. They are the primitive, two-dimensional equivalents of modular architecture that, with infinite permutations and combinations, exhaust all possibilities of a multicursal maze. They have differentiated order, attributes, and spatial meanings but they are all formed by one element like different scenarios formed by the go pieces. At the microscale, the maze is deconstructed into a single non-subdivisible unit - the double L shape, which clusters generatively into all four archetypes of maze vocabulary - the corner, the passage, the junction, and the dead end - which aggregates into a system 2 . The conventional maze generation and maze solving algorithms based on graph theory [9], matrix conversion, recursive division, dynamic programming, and search-based procedural content generation [7] are not applicable in the context of Moving Maze due to the adaptive reconfiguration of multicursal paths. Unlike Kim et al. who use the multitude of the five node conditions as input to generate a maze, we are less concerned with the quantitative attributes of the maze layout than the procedural interaction between the environment and the player, since the Moving Maze does not retain the same layout as the default stage. When the environment gains agency, the approaches to static spatial configuration mentioned above no longer apply.

\subsection{Reactive, Recursive Movement}

The environment and player agency brings opportunity for a novel design method in which we implement Blow's vision of minimal contrivance and an intelligent player [1]. In accordance with the philosophy of go, we design a moving system that operates itself according to fewest rules. In the initial state, the atomic unit (double L) is propagated with random rotations providing at least one solution path from the start to the destination. In the Moving Maze version 1.0 , the single player enters the maze and aims to navigate a way to a given destination. With each step the player moves, the system takes the player's move as an input and makes one change to the maze layout by applying implicit rule-based operations to some of the units 3. In each turn, the gamer can move in any orthogonal direction for arbitrary steps until they transgress into another unit, and the system would subsequently reconfigure by partial rotation in an adversarial mode. The player, who learns to recognize the maze's patterns of movement and picks up how to manipulate it, eventually navigates to the destination with eureka moments.

\section{PLAYER ENGAGEMENT}

In the Moving Maze, as in any video game, being engaging is a virtue and being boring is a vice. Being engaging to the player involves two intertwined concepts - active game mechanics involving interactivity and adaptability, and balanced agency between the player and the environment. The game is aptly interactive since the animated architecture serves as both an environment and an opponent. Adaptability is achieved in the Reinforced Learning scheme, as the maze auto-adjusts itself to the player's level, retaining enough challenge not to let the player take a hold too easily, but not too exhaustive of the player's intellect.

As "fun" is too broad and heterogeneous a concept, modern games need to specify the type of psychological state evoked in the player. For combat games, the psychological reward is specified as the feeling of dominance by defeating opponents; for role playing games (RPG), it is vicarious self-identification; for idle games, it is the satisfying compulsive repetition of a simple action. For the Moving Maze, positioned between puzzle and adventure, such psychological reward is the eureka moment arrived by deductive thinking and problem solving, coupled with a slight rise of adrenaline induced by the time limit. We endow the player with balanced agency defined in terms of intellectuality, intentionality, and autonomy. A slot machine produces a gameplay in which the player's complete loss of agency may surrender the player's identity during play [14]. Our Moving Maze respects the player as an intelligent person motivated by moderate cognitive challenges, as Jonathan Blow [1] concurs, in an intrigued state of mind, with a desire to explore. While a fraction of puzzle games rely on trial and error, the Moving Maze is not merely a gameplay on randomness. 

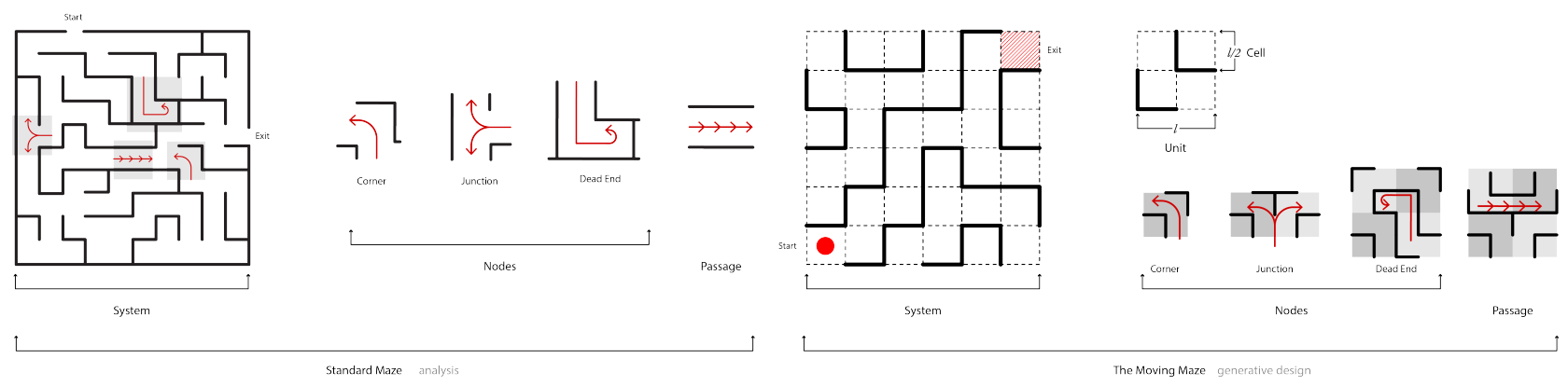

Figure 2: Based on system-unit analysis of the standard maze, we chose the double $L$ as the generative unit in appreciation of its versatility in combining into all of the constructive maze vocabulary.
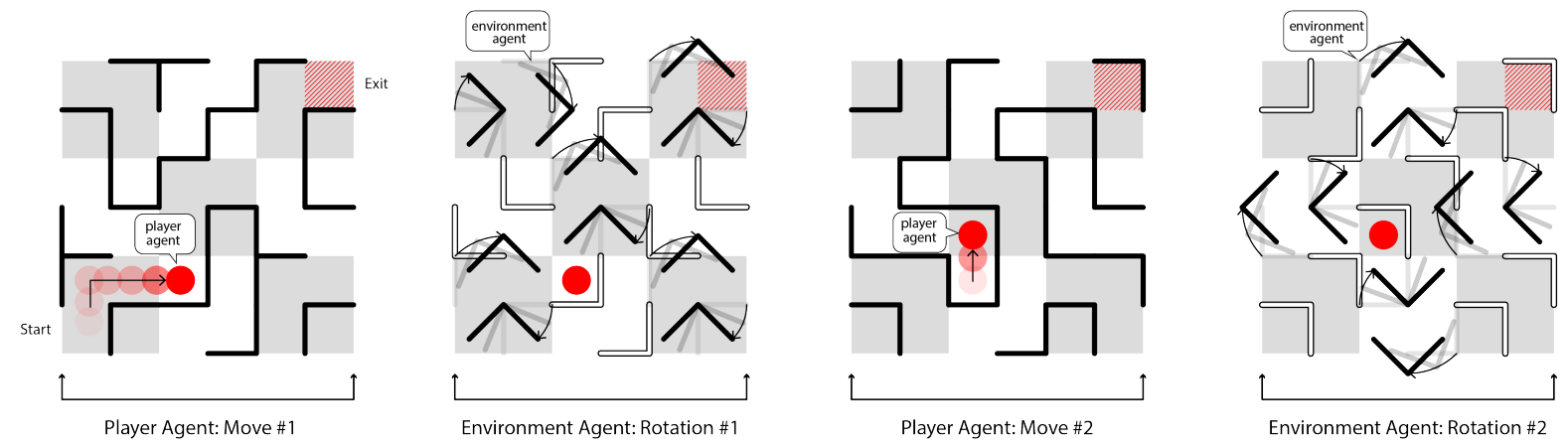

Figure 3: One move by the player agent consists of a walk in any direction. If the player's move transgressed across units, it triggers one corresponding move by the environment agent, which consists of a rotation of a diagonal set of units other than where player stands.

The game mechanics both grants autonomy and requires skills: the spatially and temporally multicursal paths requires the player's ability to think ahead and weigh different options in a problemsolving fashion, and the time limit risks wrong choices and rewards correct ones.

Synthesizing the choice nature of a maze and the movement mechanics that imposes challenge, the prototype of the game is designed on a small scale of $3^{\star} 3$ units, with simple and determinate solution pathways during any in-between static state, a measure to counterbalance the uncertainty introduced by the movement mechanics.

\subsection{Why the Moving Maze is Both a Zero-Sum Game and a Non Zero-Sum Game}

In game theory, a zero-sum game is between two binary agents whose correlations are inverse [2]. In the Moving Maze, the player agent's basic goal is to navigate to the destination while that of the environment agent (rotational walls) is to disorient the player. The opposing goals creates a zero-sum situation of the player playing against the maze. In this sense, the maze is trained as the player's opponent in chess or go, attuning to the explicit goal of destroying the player agent's solution path. But unlike AlphaGo, the ultimate goal of the Moving Maze is to immerse the human player in an engaging, rich game experience rather than defeat the human player.
The environment agent would simultaneously obstruct the human player and mitigate this adversarial action to match the level of the player to enable them a successful exit most of the time, but remain challenging to them with the probability to let them fail. Therefore, the Moving Maze is not just a human-machine zero-sum game but also a non zero-sum game, whose game mechanics is explicitly adversarial but implicitly cooperative.

\subsection{Aesthetics}

The Moving Maze drew aesthetic inspiration from legendary Chinese long-scroll painting $A$ Thousand Li of Rivers and Mountains 4. Painted by the 18-year-old Wang Ximeng in 1113, the long scroll depicts mountains on water in a dreamlike, ethereal ambience. Although much smaller in scale, the game shares the aerial view and desired ambience of the literati romanticism, and aims to convey the sentiment and elegance of $A$ Thousand $L i$ by simplistic abstraction and sensitive color selection.

\section{GENERATIVE ENVIRONMENT AND GAME DEVELOPMENT}

With rule-based real-time reconfiguration, our maze is distinguished from Kaplan's reconfigurable maze built up by modular components [5] in the temporal dimension. The Moving Maze game software 


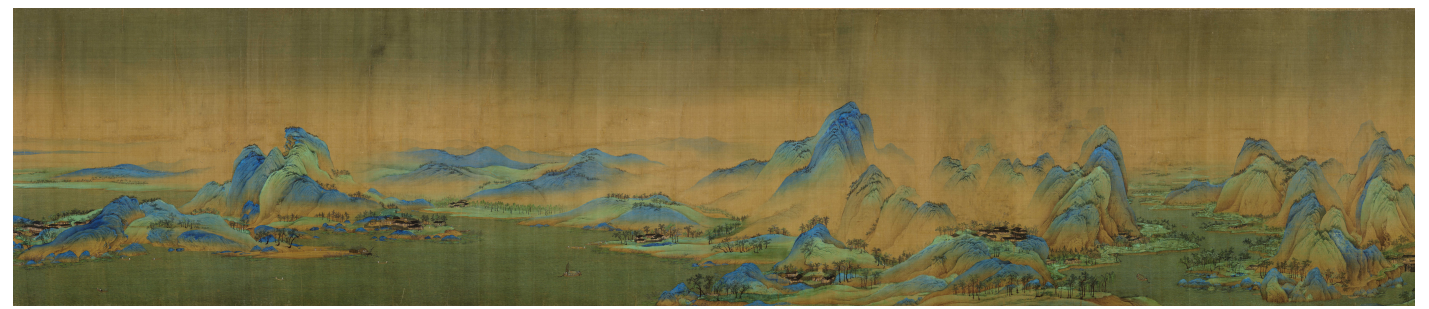

Figure 4: A section of A Thousand Li of Rivers and Mountains section. Color on silk. Palace Museum, Beijing.
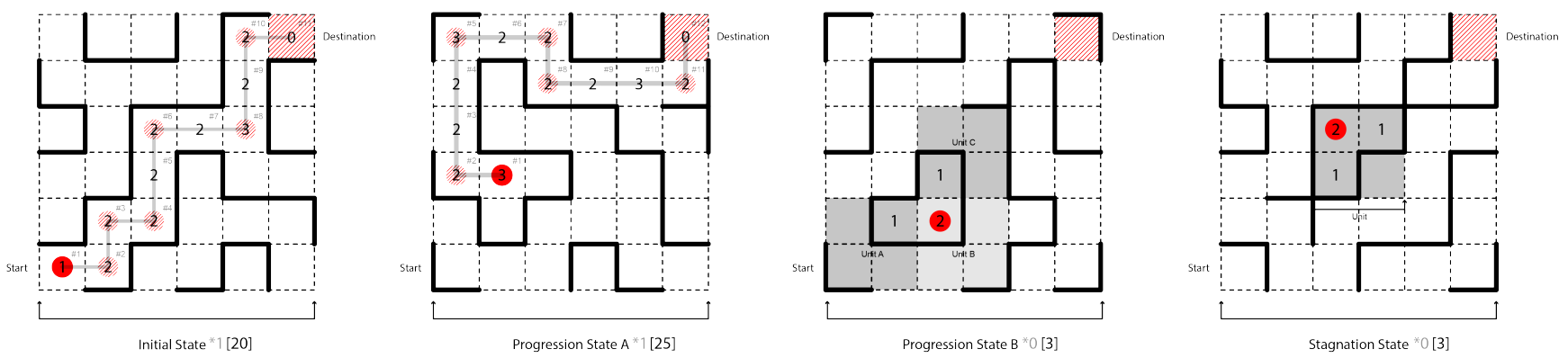

- Solution Path

Number of Solution Paths (NoSP)

Turn: the change of direction along the solution path. Number of cell ( $\mathrm{NoC}$ ) along the solution

$N$ Freedom Score (FS): the number of possible directions to move forward in a given cell. [N] Sum of Freedom Score (SoFS)

Figure 5: Game state parameters.

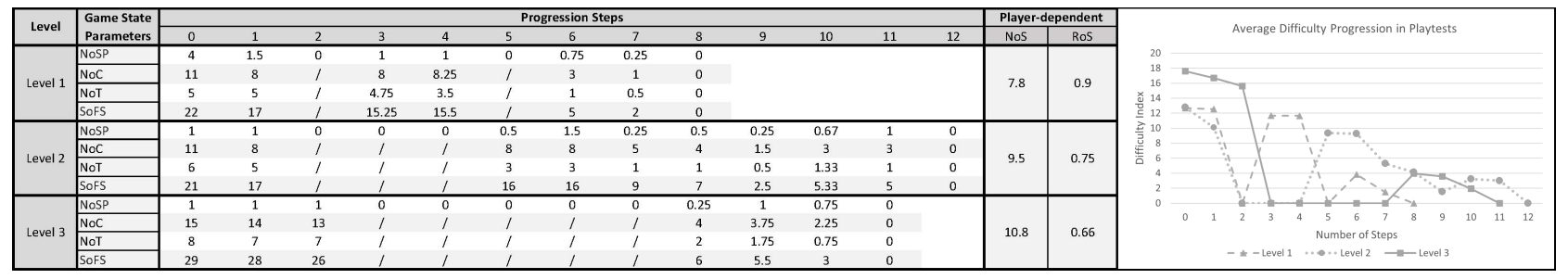

Figure 6: Average difficulty progression of three consecutive levels in playtests of 5 players

prototype was developed in Unity 3D game engine with Unity MLAgents for training purposes. The WebGL version is published at https://y-w.itch.io/moving-maze

\subsection{Difficulty Progression and Playtests}

To manage the difficulty progression in the gaming process, we define a set of parameters. Game state parameters determine the level of difficulty, measured by Difficulty Index, from the player's location to the destination along the shortest solution path. Playerdependent parameters evaluate the overall player experience.

Game state parameters5: Number of solution paths (NoSP); Number of cells (NoC), the length of the specific solution path; Number of turns (NoT), the change of direction along the solution path; Sum of freedom score (SoFS), with freedom score defined as the number of possible orthogonal directions to move forward in a given cell.
Player-dependent parameters: Number of steps (NoS) for a player to complete a solution path measured in the crossing of cells (each step consumes same amount of time); Rate of success (RoS), the probability of successful completion of a given level by a player.

We propose that the Difficulty Index (DI) of a solution path at a certain moment, from the current Location (L) to Destination (D), is a weighted sum of NoSP, NoC, NoT, and SoFS: DI (L,D) = $-a^{*} \mathrm{NoSP}+b^{*} \mathrm{NoC}+\mathrm{c}^{*} \mathrm{NoT}+\mathbf{d}^{*} \mathrm{SoFS}$

A smooth difficulty progression is quantified as an incremental increase of Difficulty Index while leveling up. It is also important to notice that these game state parameters are interdependent. To better approximate the weight of each parameter, playtests data through inverse reinforcement learning at statistically meaningful scale are needed.

To evaluate playability and player engagement, we conducted preliminary desktop playtests with five player-testers. The results 
6 (with initial weights $a=0.1, b=0.3, c=0.2, d=0.4$ and "/" indicates value not available) are shown below:

\subsection{Game Balance and Reinforcement Learning}

Game balance is, to appropriate Jaffe [4] with our lens, the finetuning of the game to be engaging, moderately challenging, and often manageable with incremental rise in difficulty. Game balancing is often a time-consuming process, and new learning algorithms have recently been used to make this process more efficient [11]. In our initial Moving Maze game prototype, there are two competing agents in the generative environment: the player agent and the environment agent. To achieve greater game balance is to achieve smoother Difficulty Index fluctuation, we propose to introduce Adversarial Reinforcement Learning [13] in this multi-agent, adversarial environment to improve competitive game mechanics by implementing Unity ML-Agents Toolkit in Unity game engine [3] to train both the player agent and the environment agent. We identify three possible training methods for future development: Train both player agent and environment agent simultaneously through imitation learning; Train player agent first through RL/imitation learning, then apply trained player agent to train environment agent through RL; Train both player agent and environment agent simultaneously through machine self-play.

\section{CONCLUSION AND FUTURE WORK}

The concept of the maze as a gameable architecture arises from its spatial potential to engender modes of play, measured on player engagement, which involves the game mechanics and balanced player agency and environment agency. Based on our component analysis of the maze's system-unit structure, a novel interactive gameable maze, rather than perfecting classic static maze with algorithms, emerges, where the maze acts as an invisible or environment agent.

Since the maze is moving, the measurements of difficulty and gameability differs from the criteria of previous mazes. Positioned between a puzzle game and an adventure game, the Moving Maze's player engagement stems from rewarding the player with eureka moments amidst cognitive challenges, and involves an interactive and adaptive game mechanics in which explicitly moves against the player agent's navigation but implicitly adjusts its intelligence to match the player for a manageable rivalry and agency balance, and is enhanced by a sensationally attractive visual style.
Through playtests and speculations, we identify some important future opportunity spaces, exploring tentatizing experimentations of game theories, more elaborate forms of cooperation, competition, and communication; emergence of new Non-player Characters (NPC) such as a second player alongside the human player, or the role reversal where the human manipulates the maze walls; room for intelligent non-player agents, such as a Minotaur that introduces risk; animated architecture in various ways expands possibilities of the environment agent, such as the growth component of a hedge maze. More modes of play are waiting to be unlocked, each of which is based on a unique understanding and setup of human relationships and the Homo Ludens' desire to game.

\section{REFERENCES}

[1] Jonathan Blow. 2011. "Video Games and the Human Condition" at Rice University. (March 2011). https://www.youtube.com/watch?v=SqFu5O-oPmU

[2] Emmanuel Guardiola and Stéphane Natkin. 2005. Game Theory and video game, a new approach of game theory to analyze and conceive game systems. In CGAMES'05, Int. Conf. on Computer Games, Angoulème, France. X, France, 166-170. https://hal.archives-ouvertes.fr/hal-01125066

[3] Nancy Iskander, Aurelien Simoni, Eloi Alonso, and Maxim Peter. 2020. Reinforcement Learning Agents for Ubisoft's Roller Champions. arXiv:2012.06031 [cs.LG]

[4] Alexander Jaffe, Alex Miller, Erik Andersen, Yun-En Liu, Anna Karlin, and Zoran Popovic. 2012. Evaluating Competitive Game Balance with Restricted Play. Proceedings of the AAAI Conference on Artificial Intelligence and Interactive Digital Entertainment 8, 1 (Oct. 2012). https://ojs.aaai.org/index.php/AIIDE/article/view/ 12513

[5] Craig S. Kaplan. 2014. The Design of a Reconfigurable Maze. In Proceedings of Bridges 2014: Mathematics, Music, Art, Architecture, Culture, Gary Greenfield, George Hart, and Reza Sarhangi (Eds.). Tessellations Publishing, Phoenix, Arizona, 167-174. Available online at http://archive.bridgesmathart.org/2014/bridges2014167.html.

[6] Radović Ljiljana Kappraff, Jay and Slavik Jablan. 2016. Meanders, Knots, Labyrinths and Mazes. Fournal of Knot Theory and Its Ramifications 25, 9 (2016).

[7] Paul Hyunjin Kim. 2019. Intelligent Maze Generation, Dissertation for the PhD at Ohio State University. (2019).

[8] Tomasz P. Pasek. 2016. Hierarchical Mazes in Psychological Research. The American fournal of Psychology 129, 4 (2016).

[9] Hans Pedersen and Karan Singh. 2006. Organic Labyrinths and Mazes. In Proceedings of the 4th International Symposium on Non-Photorealistic Animation and Rendering (Annecy, France) (NPAR '06). Association for Computing Machinery, New York, NY, USA, 79-86. https://doi.org/10.1145/1124728.1124742

[10] Jeff Saward. 2002. Magical Paths : Labyrinths and Mazes in the 21st Century. London: Mitchell Beazley.

[11] Nenad Tomasev, U. Paquet, D. Hassabis, and V. Kramnik. 2020. Assessing Game Balance with AlphaZero: Exploring Alternative Rule Sets in Chess. ArXiv abs/2009.04374 (2020).

[12] Christopher W. Totten. 2014. An architectural approach to level design (1st ed.). Boca Raton, Florida: CRC Press.

[13] William Uther and Manuela Veloso. 2003. Adversarial Reinforcement Learning, Technical report at School of Computer Science, Carnegie Mellon University. (2003).

[14] Annabel J. Wharton. 2015. Architectural agents : The delusional, abusive, addictive lives of buildings. Minneapolis, MN: University of Minnesota Press. 